\title{
miR-106b promotes cell invasion and metastasis via PTEN mediated EMT in ESCC
}

\author{
JIANXIANG ZHANG ${ }^{1 *}$, DANJIE CHEN ${ }^{2 *}$, SHUYING LIANG $^{2}$, JUN WANG $^{2}$, CAN LIU $^{2}$, \\ CAIPING NIE ${ }^{2}$, ZHENGZHENG SHAN ${ }^{2}$, LIUXING WANG ${ }^{2}$, QINXIA FAN $^{2}$ and FENG WANG ${ }^{2}$ \\ Departments of ${ }^{1}$ General Surgery and ${ }^{2}$ Oncology, The First Affiliated Hospital of Zhengzhou University, \\ Zhengzhou, Henan 450052, P.R. China
}

Received March 24, 2017; Accepted November 22, 2017

DOI: $10.3892 / \mathrm{ol} .2018 .7861$

\begin{abstract}
MicroRNA (miR)-106b serves an essential function in a variety of human cancer types, particularly in the process of invasion and metastasis. However, the function and mechanism of miR-106b in the invasion and metastasis of esophageal squamous cell carcinoma (ESCC) has remained elusive. In the present study, it was demonstrated that miR-106b was upregulated in ESCC tissues and cell lines. Furthermore, miR-106b expression in ESCC tissues was positively associated with lymphatic metastasis. Inhibition of miR-106b in EC-1 and EC9706 cells decreased not only the invasion and metastasis ability but also the proliferation ability of EC-1 and EC9706 cells. In addition, miR-106b had the ability to induce epithelial-to-mesenchymal transition (EMT) in EC-1 and EC9706 cells. In terms of the underlying mechanism, it was revealed that miR-106b promoted the invasion, metastasis and proliferation ability of EC-1 and EC9706 cells by directly targeting phosphatase and tension homolog (PTEN). Furthermore, miR-106b induced EMT in EC-1 and EC9706 cells by suppressing the expression of PTEN. In summary, the present study revealed that miR-106b contributed to invasion and metastasis in ESCC by regulating PTEN mediated EMT. Downregulation of miR-106b may be a novel strategy for preventing tumor invasion and metastasis.
\end{abstract}

\section{Introduction}

Esophageal squamous cell carcinoma (ESCC) is the most malignant lesion in China, particularly in Linzhou, Henan (1).

Correspondence to: Professor Feng Wang, Department of Oncology, The First Affiliated Hospital of Zhengzhou University, 1 Jianshe Road, Zhengzhou, Henan 450052, P.R. China

E-mail: fwang010@163.com

${ }^{*}$ Contributed equally

Key words: esophageal squamous cell carcinoma, microRNA-106b, invasion and metastasis, phosphatase and tensin homolog, epithelial-to-mesenchymal transition
Treatment of ESCC is largely useless due to the occurrence of invasion and metastasis in the early stage. However, the mechanism of invasion and metastasis of ESCC has been poorly elucidated (2).

Epithelial-to-mesenchymal transition (EMT) now is always viewed as a key event that occurs during cancer invasion and metastasis. However, current understanding of the alterations that give rise to the occurrence of EMT is limited. MicroRNAs (miRNA/miRs) are small non-coding RNA molecules that may directly regulate target gene expression by binding to their 3'-untranslated regions (3'-UTRs) $(3,4)$. Furthermore, miRNAs have been demonstrated to serve notable functions in tumor invasion and metastasis by functioning as EMT suppressors or inducers $(5,6)$. miR-106b is a type of miRNA transcribed from the miR-106b-25 cluster located on chromosome 7 (7). Yau et al (8) reported that miR-106b overexpressed in hepatocellular carcinoma cells and that the overexpression of miR-106b may promote cell migration and metastasis by activating the EMT process. Zhou et al (9) additionally demonstrated that miR-106b contributed as an EMT inducer in breast cancer. However, whether miR-106b participates in the invasion and metastasis process of ESCC by inducing EMT and the mechanism of how miR-106b induces EMT in ESCC had not been fully explored until now.

miRNAs participate in the occurrence and development of a cancer by regulating the post-transcriptional process of their target gene. Several genes have been verified to be the targets of miR-106b, including transforming growth factor- $\beta$ type II receptor, cyclin dependent kinase inhibitor $1 \mathrm{~A}$, DLC-1 Rho GTPase activating protein, signal transducer and activator of transcription 3 and mitogen-activated protein kinase 14 (7,10-12). Phosphatase and tensin homolog (PTEN), a key negative regulator of the phosphoinositide 3-kinase $(\mathrm{PI} 3 \mathrm{~K}) /$ protein kinase $\mathrm{B}(\mathrm{Akt})$ pathway, is also a direct target of miR-106b (13). One previous study had reported that PTEN served as a tumor-suppressing gene in cancer invasion and metastasis (14). Additionally, PTEN is a key factor in inducing EMT (15). However, whether miR-106b regulates the expression and activity of PTEN in ESCC has never been previously elucidated, to the best of our knowledge.

In the present study, it was determined that the expression of miR-106b was substantially higher in ESCC tissues and cell lines compared with non-tumorous tissues and cells. 
Furthermore, it was demonstrated that the upregulation of miR-106b was significantly associated with ESCC tissues lymph node metastasis and that the reduction of miR-106b expression in ESCC cell lines may inhibit cell metastasis, invasion and proliferation. Further mechanism exploration revealed that PTEN was a direct target of miR-106b. Therefore, miR-106b may participate in the invasion and metastasis of ESCC via PTEN mediated EMT.

\section{Materials and methods}

Patients. Samples of ESCC tissues and adjacent normal mucosa in paraffin-embedded blocks were collected from 32 patients who were undergoing an esophagectomy in the First Affiliated Hospital of Zhengzhou University (Henan, China). The mean age of the 32 patients (14 females and 18 males) was 60.7 \pm 8.1 years (range, 40-78). None of the patients had received radiation therapy or chemotherapy prior to surgery. All specimens were dissected, transferred and frozen immediately in liquid nitrogen at $196^{\circ} \mathrm{C}$ for RNA extraction. The present study was approved by the Ethics Committee of Zhengzhou University (Henan, China) and written informed consent was obtained from all patients prior to surgery.

Cell lines, cell culture and cell transfection. Immoral embryonic esophageal epithelium cell line SHEE and the esophageal squamous cell carcinoma cell lines EC-1 and EC9706 were all maintained at $37^{\circ} \mathrm{C}$ and $5 \% \mathrm{CO}_{2}$, in RPMI-1640 medium with $10 \%$ fetal calf serum (Hangzhou Sijiqing Bioengineering Material Co., Hangzhou, China), supplemented with $100 \mu \mathrm{g} / \mathrm{ml}$ streptomycin and $100 \mathrm{U} / \mathrm{ml}$ penicillin G. Transfection was generally performed on cells that were at $\sim 65 \%$ density. miR-106b inhibitor purchased from GE Healthcare Dharmacon, Inc. (Lafayette, CO, USA) was transfected into EC-1 or EC9706 cells by using Lipofectamine ${ }^{\circledR} 2000$ at room temperature for $6 \mathrm{~h}$ (Invitrogen; Thermo Fisher Scientific, Inc., Waltham, MA, USA). EC-1 or EC9706 cells transfected with scrambled miRNA purchased from GE Healthcare Dharmacon, Inc., or blank EC-1 or EC9706 cells were used respectively as negative or blank controls. For mRNA and protein assays, cells were all collected after $48 \mathrm{~h}$ of transfection. PTEN siRNA designed and synthesized by Zhengzhou Chuangsheng Company Co., Ltd. (Zhengzhou, China) were transfected together with miR-106b inhibitor or scrambled miRNA into EC-1 or EC9706 cells by using Lipofectamine ${ }^{\circledR} 2000$ at room temperature for 6 h. EC-1 or EC9706 cells transfected individually with PTEN siRNA or blank EC-1 cells were used as the control.

$R N A$ extraction and reverse transcription-quantitative polymerase chain reaction $(R T-q P C R)$ assay. Total RNA was extracted using either an miRNeasy kit (Qiagen, Inc., Valencia, CA, USA) or TriReagent (Sigma-Aldrich; Merck KGaA, Darmstadt, Germany) at room temperature for $10 \mathrm{~min}$ according to the manufacturer's protocols from tissues lysates or cell samples and reverse transcribed to cDNA with TaqMan ${ }^{\circledR}$ miRNA Reverse Transcription kit (Applied Biosystems; Thermo Fisher Scientific, Inc.) or tranScript First-strand cDNA Synthesis SuperMix kit (Qiagen, Inc.). The relative expression levels of miR-106b were determined using the TaqMan ${ }^{\circledR}$ MicroRNA Assay
(Applied Biosystems; Thermo Fisher Scientific, Inc.) protocol specific for miR-106b and were normalized to that of internal control U6 by using the $2^{-\Delta \Delta \mathrm{Cq}}$ method (16) While the relative expression levels of PTEN were determined using the STBR Premix Ex Taq ${ }^{\mathrm{TM}}$ kit (Qiagen GmbH, Hilden, Germany) with the following primers: PTEN sense, ACTGGACCACCTAAT TGCTGT and antisense, GCAAACTTTTACACTGGCAGC; E-cadherin forward, CTCAAAGCCCAGAATCCCCA and reverse, CGGTTTTCTGTGCACACCTG; vimentin forward, TCCGCACATTCGAGCAAAGA and reverse, ATTCAA GTCTCAGCGGGCTC. The relative amount of mRNA was normalized by using $\beta$-actin. Experiments were performed in triplicate. The thermal cycle for miRNA was: $94^{\circ} \mathrm{C}$ for $3 \mathrm{~min}$; $95^{\circ} \mathrm{C}$ for $15 \mathrm{sec} ; 65^{\circ} \mathrm{C} 40$ times for $40 \mathrm{sec}$; and $62^{\circ} \mathrm{C}$ for $40 \mathrm{sec}$. For others the thermal cycle was: $95^{\circ} \mathrm{C}$ for, $10 \mathrm{~min} ; 94^{\circ} \mathrm{C}$ for $40 \mathrm{sec} ; 56^{\circ} \mathrm{C}$ for $35 \mathrm{sec} ; 72^{\circ} \mathrm{C} 30$ times for $60 \mathrm{sec}$; and $72^{\circ} \mathrm{C}$ for $10 \mathrm{~min}$.

Western blot assay. The protein contents of tissues or cell lysates were measured using a BCA kit (Beyotime Institute of Biotechnology, Shanghai, China). Then suitable amount of total protein of each sample $(10-20 \mu \mathrm{g})$ was separated by $10 \%$ SDS-PAGE. Following vertical electrophoretic separation, proteins were shifted to polyvinylidene fluoride (PVDF) membranes by wet transfer. Subsequently, the PVDF membranes were blocked with $5 \%$ fat-free milk for $1 \mathrm{~h}$ at room temperature, and then incubated with rabbit anti-PTEN antibodies (dilution 1:1,000; cat no. AF1426) rabbit anti-E-cadherin (dilution 1:500; cat. no. AF0138) and rabbit anti-vimentin (dilution 1:1,000; cat. no. AF1975) (all from Beyotime Institute of Biotechnology) at $4^{\circ} \mathrm{C}$ overnight. Following washing the membranes for three times in tris-buffered saline with Tween-20 (TBST), the membranes were incubated with HRP-conjugated secondary antibody (dilution 1:5,000; cat. no. A0208; Beyotime Institute of Biotechnology) at room temperature for $1 \mathrm{~h}$. Subsequent to washing three times with TBST again, exposure and development were performed using an ECL Chemiluminescence Detection kit (GE Healthcare Life Sciences, Little Chalfont, UK) and the densities were measured using the GS-900 ${ }^{\text {TM }}$ Calibrated Densitometer with ImageLab 4.1 software from Bio-Rad Laboratories, Inc. (Hercules, CA, USA). All of the assays were performed in triplicate.

Boyden chamber invasion assay. A Boyden chamber invasion assay was performed as previously described (17). Briefly, the upper chamber was coated with Matrigel were filled with EC-1 or EC9706 cells suspended in serum-free RPMI-1640 medium, while the lower chamber was loaded with RPMI-1640 medium with $10 \%$ fetal calf serum. EC-1 or EC9706 cells were allowed to invade through the filter for $48 \mathrm{~h}$ at $37^{\circ} \mathrm{C}$ and the cells on the upper sides were removed using cotton swabs. The cells that invaded to the lower side were subjected to H\&E staining at room temperature for $7 \mathrm{~min}$ and calculated in five random fields per membrane under inverted microscopy at x200 magnification. Migration experiments were performed in triplicate.

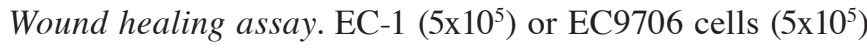
were plated into 12 -well plates and grew to $70 \%$ confluence. Then, a constant wide wound $(1 \mathrm{~mm})$ was created using a plastic tip on monolayer cultured EC-1 or EC9706 cells. A 
total of $48 \mathrm{~h}$ after the migration of EC-1 or EC9706 cells, the average migrating distance was measured under an inverted microscope and photographed at x200 magnification.

Cell Counting kit-8 (CCK-8) assay. The proliferation ability of EC-1 or EC9706 cells was measured using a CCK-8 kit according to the manufacturer's instructions. In briefly, EC-1 or EC9706 cells were seeded into 96-well plates at a density of $5 \times 10^{3}$ cells/well and incubated for $48 \mathrm{~h}$ at room temperature. Subsequently, $10 \mu \mathrm{l}$ CCK-8 solution was added to the well and cultured for another $1 \mathrm{~h}$ at room temperature. The optical density was calculated by measuring the absorbance at $450 \mathrm{~nm}$. All of the experiments were performed in triplicate.

Target prediction. The prediction of the 3'UTR of PTEN as a binding target of miR-106b was checked by using PicTar (pictar.mdc-berlin.de) and Targetscan (www.targetscan.org).

Luciferase activity assay. Pmir-REPORT system (Promega Corporation, Madison, WI, USA) was used to determine whether PTEN was a direct target of miR-106b. 3'UTR of the PTEN gene was amplified, sub-cloned into the pmirGLO luciferase reporter vector and amplified in DH5 $\alpha$. The resulting reporter vector was designated as pmirGLO-PTEN-WT. A site-directed mutagenesis kit (Stratagene; Agilent Technologies, Inc., Santa Clara, CA, USA) was used to produce the mutant type of PTEN 3'UTR luciferase reporter vector (pmirGLO-PTEN-MUT), according to the manufacturer's protocol. The luciferase reporter vectors were verified by sequencing. miR-106b inhibitor or scrambled miRNA, $100 \mathrm{ng}$ pmirGLO-PTEN-WT or pmirGLO-PTEN-MUT was co-transfected into EC-1 cells in 6-well plates using Lipofectamine ${ }^{\circledR} 2000$ at room temperature. After 48 h, luciferase activity was measured using a Dual-Luciferase Reporter Assay system (Promega Corporation).

Statistical analysis. Statistical analysis was performed using SPSS 17.0 (SPSS, Inc., Chicago, IL, USA). Multi-group comparisons were performed using a one-way analysis of variance test with a least significant difference test used for post-hoc comparisons. $\mathrm{P}<0.05$ was considered to indicate a statistically significant difference. And the data were presented as the mean \pm the standard deviation.

\section{Results}

miR-106b was upregulated in ESCC tissues and cell lines. To evaluate the expression levels of miR-106b in ESCC tissues and adjacent normal mucosa tissues, RT-qPCR was performed. The results revealed that the expression levels of miR-106b in ESCC tissues were significantly increased compared with those in adjacent normal mucosa tissue $(\mathrm{P}<0.01$; Fig. $1 \mathrm{~A})$. miR-106b has been reported to serve a notable function in cancer invasion and metastasis, thus the association between miR-106b and the clinicopathological parameters of patients with ESCC was analyzed. As was expected, the upregulation of miR-106b was significantly associated with lymph node metastasis $(\mathrm{P}<0.05$; Fig. 1B). Then, the expression levels of miR-106b were examined in ESCC cell lines EC-1 and EC9706. They were revealed to be significantly higher, compared with that

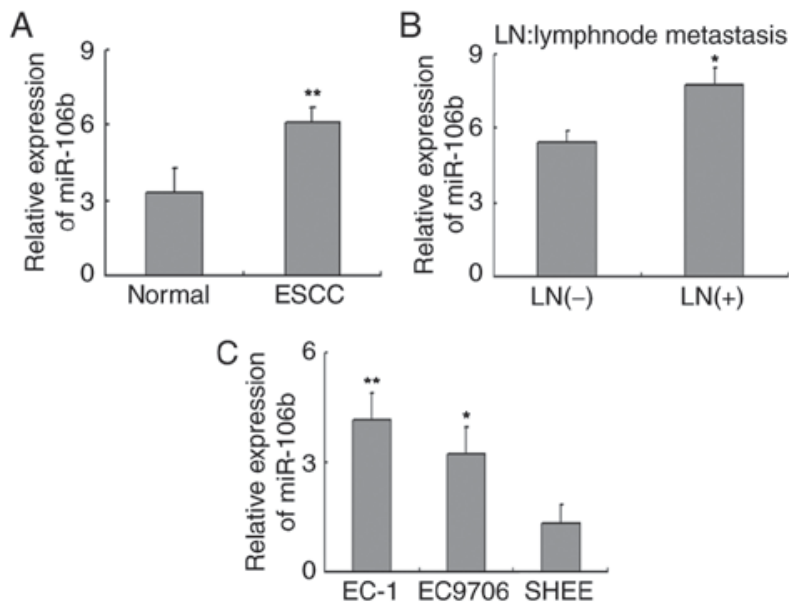

Figure 1. Upregulation of miR-106b in ESCC tissues and cell lines. (A) Expression level of miR-106b in ESCC tissues and adjacent normal mucosal tissues were analyzed by reverse transcription-quantitative polymerase chain reaction. The results revealed that miR-106b was significantly upregulated in ESCC tissues compared with that in adjacent normal mucosa tissues. ${ }^{* *} \mathrm{P}<0.01$ vs. normal tissues. (B) miR-106b was revealed to be upregulated in ESCC tissues with lymph node metastasis compared with ESCC tissues without lymph node metastasis. ${ }^{*} \mathrm{P}<0.05$ vs. ESCC tissues without lymph node metastasis. (C) Expression level of miR-106b in ESCC cell lines EC1 and EC9706 were significantly upregulated compared with that in the immortal embryonic esophageal epithelium cell line SHEE. ${ }^{*} \mathrm{P}<0.05$ and ${ }^{* *} \mathrm{P}<0.01$ vs. SHEE. ESCC, esophageal squamous cell carcinoma; miR, microRNA.

in the immortal embryonic esophageal epithelium cell line SHEE $(\mathrm{P}<0.05)$. These findings suggest that miR-106b may promote tumorigenesis in ESCC (Fig. 1C).

Downregulation of miR-106b expression may inhibit the invasion, metastasis and proliferation ability of ESCC cells. To further elucidate the function of miR-106b in ESCC invasion and metastasis, miR-106b inhibitor was transfected into EC-1 or EC9076 cells to investigate the effects of the downregulation of miR-106 on the invasion and metastasis ability of ESCC cell lines. EC-1 cells transfected with scrambled miRNA and vacant EC-1 cells were used as negative and blank controls, respectively. The results of RT-qPCR revealed that miR-106b inhibitor significantly decreases miR-106b expression compared with those in control groups $48 \mathrm{~h}$ after transfection in $\mathrm{EC}-1$ cells $(\mathrm{P}<0.01$; Fig. $2 \mathrm{~A})$.

Then, the function of miR-106b in ESCC cells was evaluated by measuring the cell invasion and metastasis ability of EC-1 cells which were transfected with miR-106b inhibitor. EC-1 cells transfected with scrambled miRNA or vacant EC-1 cells were used as negative and blank controls, respectively. Compared with the control groups, the number of EC-1 cells transfected with miR-106b inhibitor that traversed the Matrigel were significantly lower ( $\mathrm{P}<0.05$; Fig. $2 \mathrm{~B})$. The results of a wound-healing assay further indicated the function of miR-106b in the invasion and metastasis of EC-1 cells. The wound healing ability of EC-1 cells transfected with miR-106b inhibitor was significantly decreased compared with those in control groups ( $\mathrm{P}<0.01$; Fig. $2 \mathrm{C}$ ).

The increase of the invasion and metastasis ability of cancer cells is usually closely associated with an increase of the proliferation ability. Thus, the effects of miR-106b inhibitor on the proliferation ability of EC-1 cells were examined by using 
EC-1
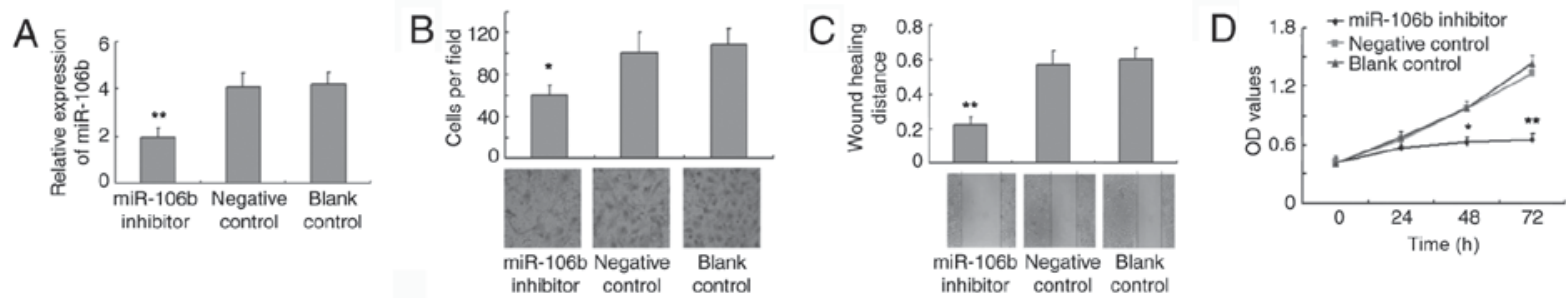

EC9706
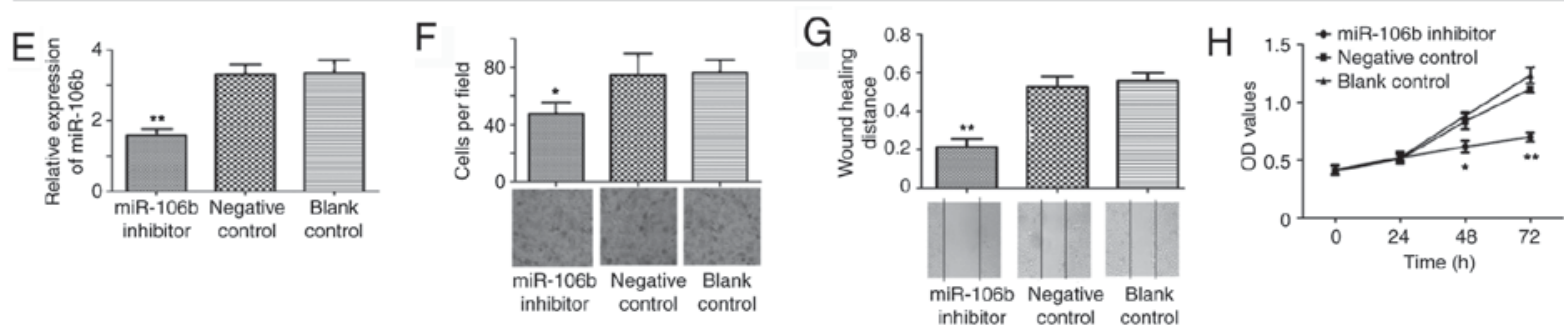

Figure 2. Downregulation of miR-106b inhibited the invasion, metastasis and proliferation ability of esophageal squamous cell carcinoma cells. miR-106b inhibitor decreased the expression level of miR-106b in (A) EC-1 cells significantly compared with that in cells transfected with scrambled miRNA or vacant cells. Results of a Matrigel assay revealed that the downregulation of miR-106b significantly inhibited the number of (B) EC-1 cells that invaded through the membrane compared with control cells (x200 magnification). Downregulation of miR-106b also significantly decreased the wound healing distance of (C) EC-1 cells compared with control cells (x200 magnification). A Cell Counting Kit-8 assay was used to examine the proliferation ability of (D) EC-1, 24, 48 and $72 \mathrm{~h}$ after transfection with a miR-106b inhibitor. EC-1 transfected with scrambled miRNA or vacant EC-1 or EC9706 cells were used as negative or blank controls, respectively. miR-106b inhibitor decreased the expression level of miR-106b in (E) EC9706 cells significantly compared with that in cells transfected with scrambled miRNA or vacant cells and in addition, the (F) invasion, $(\mathrm{G})$ metastasis and (H) proliferation of EC9706 cells transfected with miR-106b were also decreased notably compared with control groups. ${ }^{*} \mathrm{P}<0.05$ and ${ }^{* *} \mathrm{P}<0.01$ vs. negative or blank controls. miR, microRNA; OD, optical density.

a CCK-8 assay. The results revealed that the downregulation of miR-106b inhibited cell proliferation significantly compared with control groups at 48 and $72 \mathrm{~h}$ (Fig. 2D), indicative that the expression change of miR-106b may alter the proliferation of ESCC cells.

Then similar results were obtained in EC9706 cells (Fig. 2E-H). Thus, it may be concluded that miR-106b is an important factor in the invasion and metastasis process of ESCC cells.

Downregulation of miR-106b expression could inhibit the induction of EMT in ESCC cells. Previous evidence has reported that miRNAs promote invasion and metastasis through mediating EMT (18). In order to evaluate the function of miR-106b in inducing EMT in ESCC, we examined the mRNA and protein expression of the epithelial marker E-cadherin and the mesenchymal marker vimentin in EC-1 cells. As presented in Fig. 3A and B, with the downregulation of miR-106b in EC-1 cells, the mRNA and protein expression levels of E-cadherin were significantly increased compared with those in control groups $(\mathrm{P}<0.05)$, whilst the expression levels of vimentin were significantly decreased compared with the control groups $(\mathrm{P}<0.05)$. Additionally, similar results were revealed in EC9706 cells (Fig. 3C and D). Thus, miR-106b was concluded to be capable of advancing invasion and metastasis by regulating EMT in ESCC cells. Due to its predominant function in ESCC EMT, miR-106b may be used as a target to limit EMT-associated invasion and metastasis.

PTEN was directly targeted by miR-106b via the 3'-UTR. Although the major function of miR-106b in the occurrence and development of ESCC had already been elucidated, the mechanism by which miR-106b promoted invasion and metastasis had yet to be determined in ESCC cell lines. PTEN, a tumor suppressor, is known to be a negative regulator of the PI3K-Akt pathway. Loss or downregulation of PTEN has been identified in numerous different cancer types, including ESCC, and has been revealed to be associated with the invasive properties of cancer cells. By using PicTar and TargetScan software, miR-106b was revealed to have a putative biding site in the 3'-UTR of PTEN. In order to validate whether PTEN was a functional target of miR-106b, miR-106b inhibitor or scrambled miRNA was co-transfected with pmirGLO-PTEN-WT or pmirGLO-PTEN-MUT in ESCC cells. As presented in Fig. 4A, ESCC cells with miR-106b inhibitor and pmirGLO-PTEN-WT co-transfection presented a significant increase in reporter activity compared with ESCC cells with scrambled miRNA and pmirGLO-PTEN-WT co-transfection $(\mathrm{P}<0.05)$. Furthermore, ESCC cells co-transfected with miR-106b inhibitor and pmirGLO-PTEN-MUT or scrambled miRNA with pmirGLO-PTEN-MUT demonstrated no statistically significant change in reporter activity (Fig. 4A). Thus, the luciferase activity of the wild type PTEN-3'-UTR but not the mutated PTEN-3'-UTR reporter was reduced by miR-106b in ESCC cells. In order to further verify the regulatory effects of miR106b on PTEN, RT-qPCR and western blot analysis were used to check the expression levels of PTEN. The results revealed that with the downregulation of miR-106b in EC-1 or EC9706 cells, the mRNA and protein expression of PTEN were significantly increased $(\mathrm{P}<0.05$; Fig. 4B-E). However, the mRNA and protein expression of PTEN in EC-1 or EC9706 cells transfected with scrambled miRNA demonstrated no 
EC-1

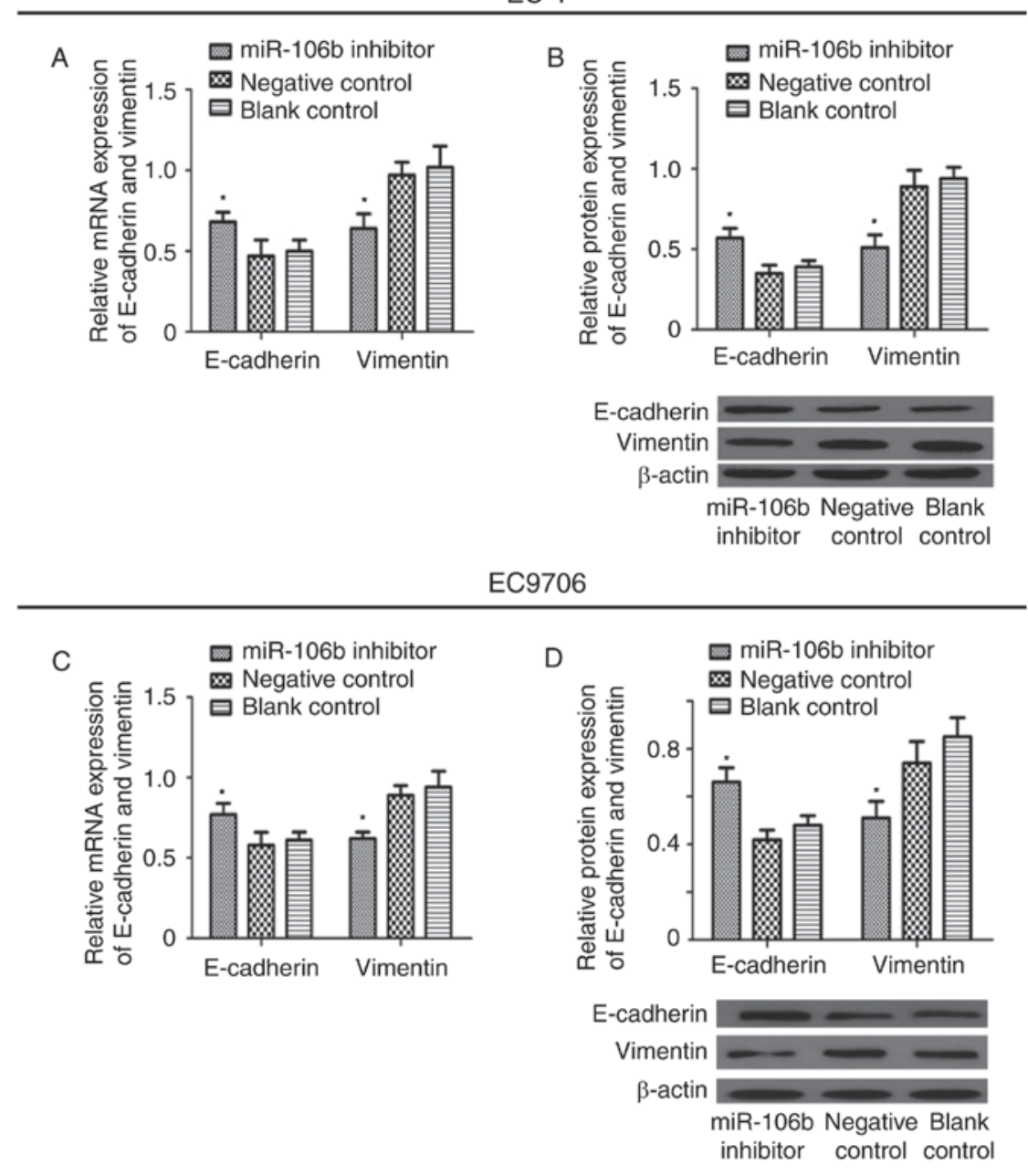

Figure 3. Downregulation of miR-106 inhibited the occurrence of epithelial-mesenchymal transition in esophageal squamous cell carcinoma cells. Reverse transcription-quantitative polymerase chain reaction and a western blot assay were used to detect the (A) mRNA and (B) protein expression levels of E-cadherin and vimentin in EC-1 cells, and the (C) mRNA and (D) protein expression levels of E-cadherin and vimentin in EC9706 cells. Results revealed that the mRNA and protein expression levels of E-cadherin were promoted in the presence of an miR-106b inhibitor compared with those in control groups, whilst the mRNA and protein expression levels of vimentin were reduced in EC-1 or EC9706 cells treated with an miR-106b inhibitor compared with control groups. ${ }^{*} \mathrm{P}<0.05$ vs. the negative or blank controls. miR, microRNA; E, epithelial.

significant difference compared with vacant EC-1 or EC9706 cells (Fig. 4B-E). These results indicate that miR-106b may regulate the expression of PTEN in ESCC cells.

Downregulation of PTEN partially increased the invasion, metastasis and proliferation ability of ESCC cells. PTEN has been reported to participate in invasion and metastasis (19). In order to evaluate whether miR-106b may participate in the invasion and metastasis of ESCC by regulating the expression of PTEN, PTEN siRNA was chemically synthesized and co-transfected into EC-1 or EC9706 cells with miR-106b inhibitor. PTEN siRNA co-transfected with scrambled miRNA, PTEN siRNA transfected into EC-1 or EC9706 cells alone and vacant EC-1 or EC9706 cells were used as the controls. In EC-1 cells, the expression levels of PTEN were significantly lower in the PTEN siRNA transfected group compared with the untransfected group $(\mathrm{P}<0.01$; Fig. 5A). Additionally, the expression levels of PTEN were significantly lower in the PTEN siRNA and miR-106b inhibitor co-transfected group compared with the miR-106b inhibitor alone transfected group $(\mathrm{P}<0.05$; Fig. 5A). A Boyden chamber invasion assay and a wound healing assay were used again to analyze the invasion and metastasis ability of EC-1 cells between different groups. A significant difference was identified in the PTEN siRNA and miR-106b inhibitor transfected group in EC-1 cells in invasion and metastasis, compared with the miR-106b inhibitor alone transfected group $(\mathrm{P}<0.05$; Fig. 5B and $\mathrm{C})$. In addition, the downregulation of PTEN may partially rescue the proliferation ability of EC-1 or EC9706 cells evaluated by using a CCK-8 assay, as a significant difference was identified in the PTEN siRNA transfected group $(\mathrm{P}<0.01)$ and the PTEN siRNA and miR-106b inhibitor transfected group $(\mathrm{P}<0.05)$ in EC-1 cells (Fig. 5D). In EC9706 cells, downregulation of PTEN also rescued the invasion and metastasis of EC9706 cells inhibited by miR-106b inhibitor, the only difference was that no significant difference was revealed in the proliferation ability of EC9706 cells (Fig. 5E-H). Altogether, miR-106b is potentially a key regulator in the occurrence and development process of ESCC that may occur via regulating the expression of PTEN.

Downregulation of PTEN partially rescued the induction of the EMT of ESCC cells. miRNAs modulate EMT by impacting the 

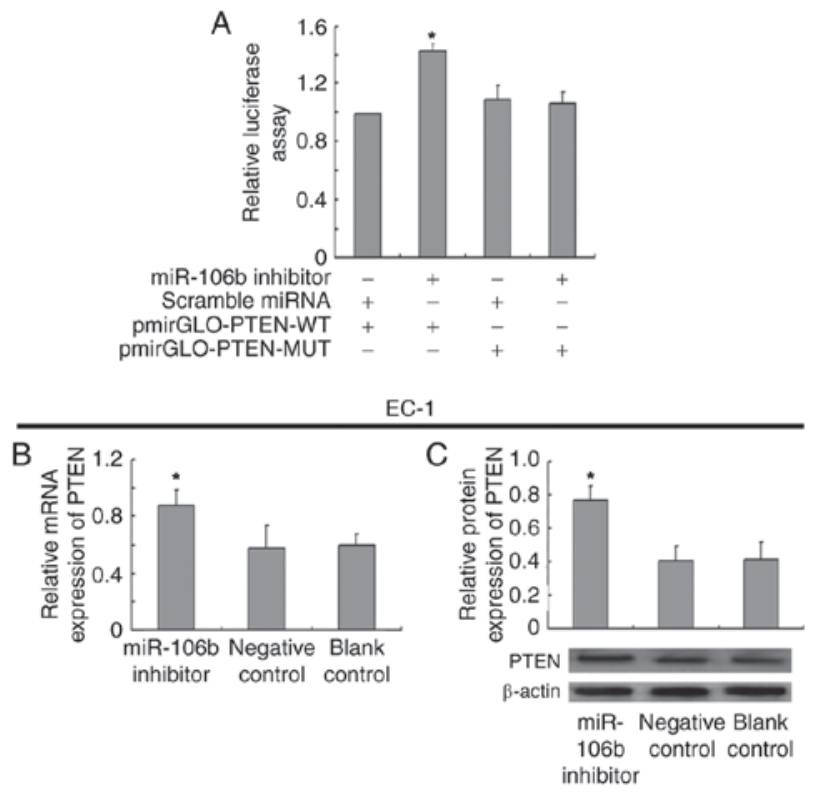

EC9706
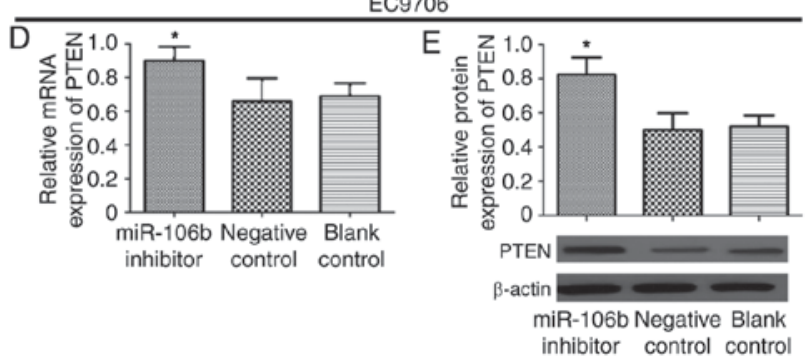

Figure 4. PTEN was directly targeted by miR-106b via the 3'-UTR. (A) Luciferase activity assay was used to determine the reporter activity in cells co-transfected with either miR106b inhibitor or scramble miRNA, and either pmirGLO-PTEN-WT or pmirGLO-PTEN-MUT. ${ }^{*}$ P $<0.05$ vs. the scramble miRNA and pmirGLO-PTEN-WT co-transfected cells. Subsequently, the relative (B) mRNA and (C) protein expression levels of PTEN in EC-1 cells with or without miR-106b inhibitor were determined. Additionally, the relative (D) mRNA and (E) protein expression levels of PTEN in EC9706 cells with or without miR-106b inhibitor were determined. $\beta$-actin was used as an internal control. " $\mathrm{P}<0.05$ vs. the control groups. PTEN, phosphatase and tensin homolog; UTR, untranslated region; miRNA/miR, microRNA.

expression of receptors, signaling pathways and specific ligands. PTEN serves a notable function in tumor invasion and migration, and its deletion and mutation is also present in numerous different cancer types and is associated with EMT. As had been previously suggested, miR-106b regulates the expression of PTEN by binding to its promoter. However, whether PTEN contributes to the EMT induced by miR-106b in ESCC had yet to be elucidated. In the present study, it was revealed that downregulation of PTEN in EC-1 or EC9706 cells partially rescued the EMT inhibited by decreased expression of miR-106b. The mRNA and protein expression levels of E-cadherin in EC-1 cells were significantly downregulated and levels of vimentin were significantly upregulated in cells transfected with PTEN siRNA alone compared with untransfected cells $(\mathrm{P}<0.01)$ and in cells transfected with PTEN siRNA and miR-106b inhibitor compared with cells transfected with miR-106b inhibitor alone $(\mathrm{P}<0.05$; Fig. 6A and $\mathrm{B})$. Identical results were revealed in EC9706 cells (Fig. 6C and D). Collectively, the results of the present study demonstrate that miR-106b induces EMT via suppressing the expression of PTEN.

\section{Discussion}

miRNAs, small non-coding RNAs of 22 nucleotides in length, have been demonstrated to be associated with the occurrence and development of human cancer types where they may function as tumor suppressors or oncogenes (3-6,20,21). Previous studies have demonstrated the abnormal expression of a number of types of miRNAs in ESCC $(22,23)$. miR-106b is a member of the miR-106b-25 cluster and it has been demonstrated to be upregulated in a number of different types of cancer, including prostate cancer, hepatocellular cancer, gastric cancer, laryngeal carcinoma and breast cancer (24-28). However, its function and mechanism in ESCC have remained unclear.

In the present study, the expression levels of miR-106b in ESCC tissues were revealed to be significantly higher compared with in adjacent normal mucosa. Consistent with the data that was investigated in ESCC tissues, it was further verified that the expression levels of miR-106b in the ESCC cell lines EC-1 and EC9706 were also significantly higher compared with in the immoral embryonic esophageal epithelium cell line SHEE, suggesting that the upregulation of miR-106b may promote the occurrence of ESCC. Furthermore, significant associations were also identified between the expression levels of miR-106b and advanced stage and lymph node metastasis, indicating that the expression of miR-106b also served a notable function in ESCC invasion and metastasis.

To further elucidate the function of miR-106b in the occurrence and development of ESCC, a miR-106b inhibitor and scrambled miRNA were synthesized individually and transfected into EC-1 or EC9706 cells using Lipofectamine ${ }^{\circledR} 2000$ for $48 \mathrm{~h}$, and vacant EC-1 or EC9706 cells were used as the blank control. As expected, the expression levels of miR-106b decreased significantly in EC-1 or EC9706 cells transfected with miR-106b inhibitor compared with those transfected with scrambled miRNA or vacant EC-1 or EC9706 cells. With the drop of the expression of miR-106b, the invasion, metastasis and proliferation ability of EC-1 or EC9706 cells also dropped significantly compared with those transfected with scrambled miRNA and vacant EC-1 or EC9706 cells. These results further validate the hypothesis that miR-106b may function as an oncogene and serves an important function in the promotion of invasion, metastasis and proliferation in ESCC cells. EMT serves an integral function in cancer metastasis and emerging evidence reveals that miRNAs are involved in cancer metastasis by regulating EMT (29). In the present study, it was demonstrated that by the downregulation of miR-106b, the mRNA and protein expression of E-cadherin were increased significantly whilst the expression level of vimentin was decreased significantly. Therefore, it was concluded that miR-106b induces EMT in ESCC cells, and miR-106b potentially participates in the metastasis process of ESCC cells by inducing EMT.

PTEN, in previous years, has been widely viewed as a tumor suppressor gene, which regulates survival, growth, invasion, metastasis and proliferation $(30,31)$. PTEN may regulate PI3K signaling by dephosphorylating the lipid signaling intermediate phosphatidylinositol (3,4,5)-trisphosphate, and a clear link between the PI3K pathway and cancer was established in the 1980s (32). miRNA always operated by binding to the 3'UTRs of their target genes and a number of previous reports 
EC-1

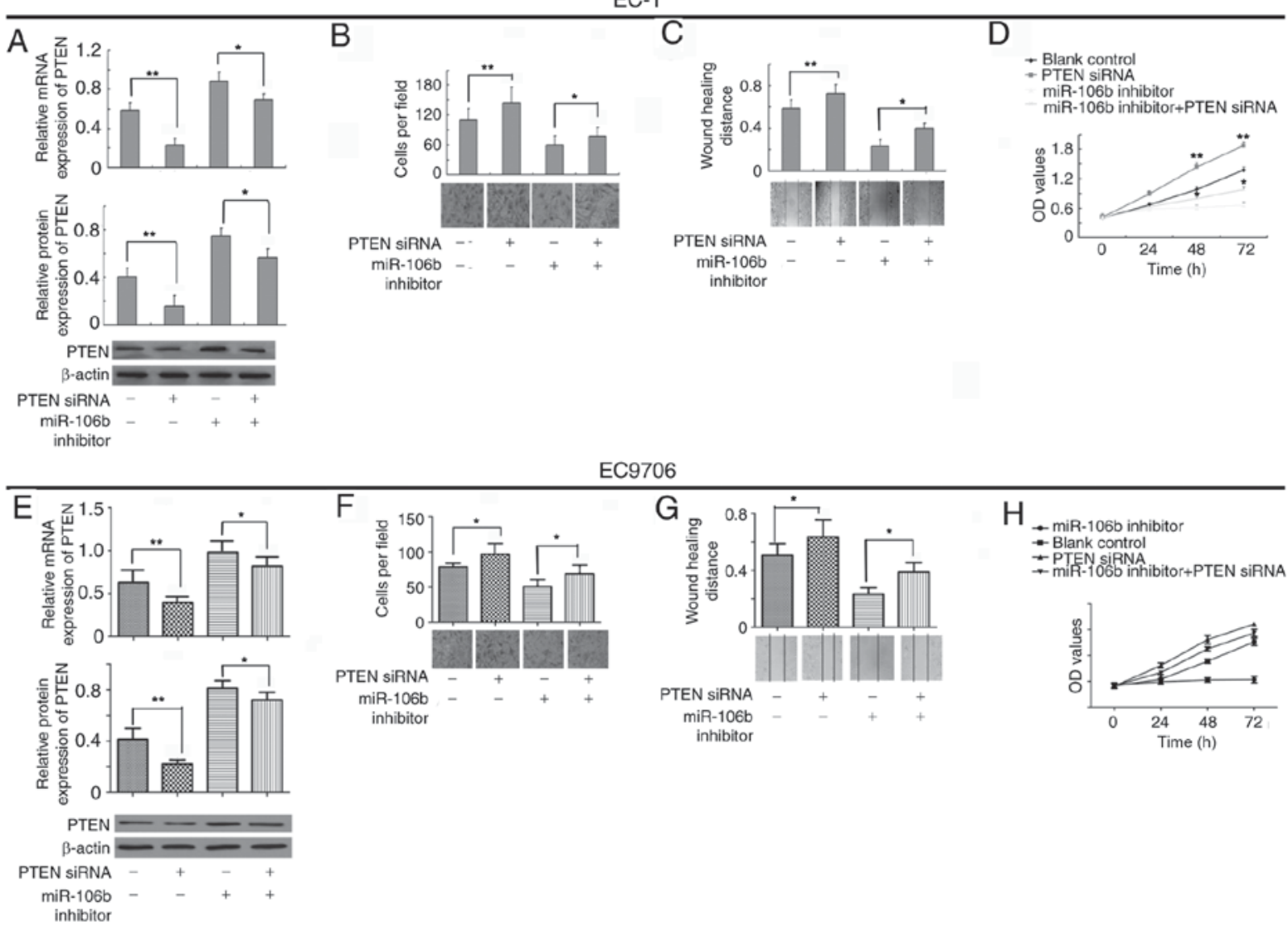

Figure 5. Downregulation of PTEN partially increased the invasion, metastasis and proliferation ability of esophageal squamous cell carcinoma cells. Downregulation of PTEN may inhibit the mRNA and protein expression level of PTEN increased by miR-106 inhibitor in (A) EC-1 cells. " $\mathrm{P}<0.05$ vs. miR-106b inhibitor alone transfected cells; ${ }^{* *} \mathrm{P}<0.01$ vs. untransfected cells. The invasion of (B) EC-1 cells, the metastasis of (C) EC-1 cells, and the proliferation of (D) EC-1 cells were examined. ${ }^{*} \mathrm{P}<0.05$ and ${ }^{* *} \mathrm{P}<0.01$ vs. untransfected cells. (E) In EC9706 cells, the downregulation of PTEN additionally inhibited the mRNA and protein expression level of PTEN increased by miR-106 inhibitor. " $\mathrm{P}<0.05$ vs. miR-106b inhibitor alone transfected cells. ${ }^{* *} \mathrm{P}<0.01$ vs. untransfected cells. The (F) invasion, $(\mathrm{G})$ metastasis and $(\mathrm{H})$ proliferation of EC9706 cells were also examined. "P<0.05 and ${ }^{* *} \mathrm{P}<0.01$ vs. untransfected cells. PTEN, phosphatase and tensin homolog; miR, microRNA; OD, optical density.

EC-1

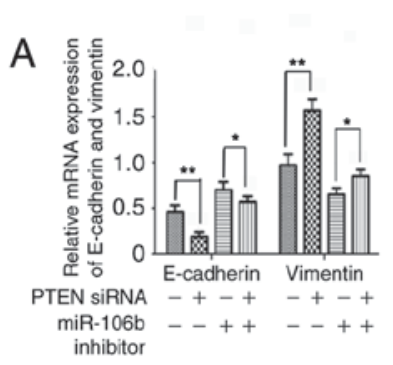

$\mathrm{B}$

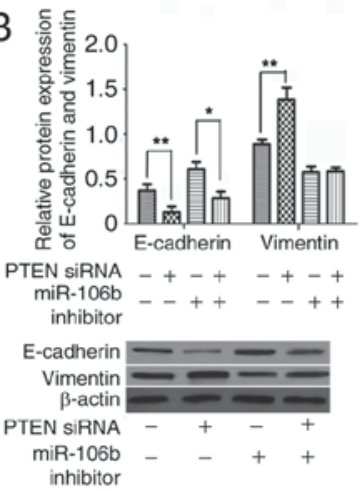

EC9706

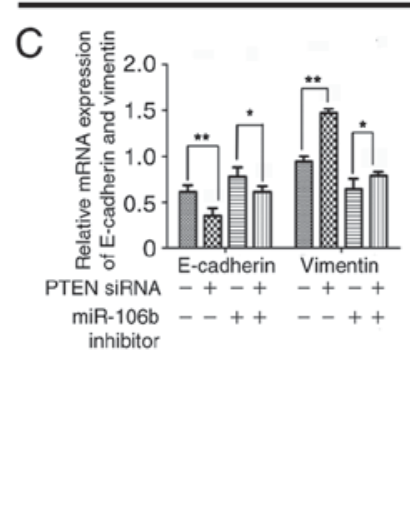

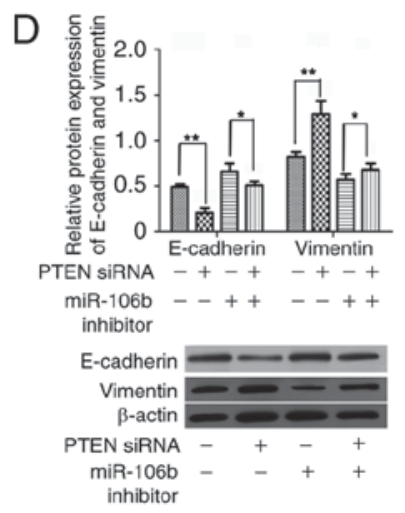

Figure 6. Downregulation of PTEN partially rescued epithelial-mesenchymal transition inhibited by the lower expression of miR-106b. Results of reverse transcription-quantitative polymerase chain reaction and western blot analysis were used to reveal the (A) mRNA and (B) protein expression levels of E-cadherin and vimentin in EC-1 cells and the (C) mRNA and (D) protein expression levels of E-cadherin and vimentin in EC9706 cells that were untransfected, transfected with either PTEN siRNA or miR-106b inhibitor alone, or co-transfected. ${ }^{* *} \mathrm{P}<0.01$ vs. untransfected cells; " $\mathrm{P}<0.05$ vs. cells transfected with miR-106b inhibitor alone. PTEN, phosphatase and tensin homolog; miR, microRNA; si, small interfering; E, epithelial.

have confirmed that miR-106b serves its part in colorectal cancer, gastric cancer and hepatocellular carcinoma (33,34). In the present study, it was demonstrated that miR-106b inhibition may result in the increased expression of PTEN in ESCC cells compared with those in control cells. Using cell transfection and luciferase reporter assay, it was additionally revealed that PTEN was a direct target of miR-106b. It was revealed that the depletion of PTEN by siRNA in EC-1 or EC9706 cells 
transfected with miR-106b inhibitor may partially reverse the effects on invasion, metastasis and proliferation caused by miR-106b downregulation. Furthermore, the depletion of PTEN partially rescued EMT inhibited by miR-106b inhibitor. These results further demonstrated that miR-106b promoted the occurrence and development of ESCC via targeting PTEN mediated EMT.

To conclude, the results of the present study suggest that miR-106b was upregulated in ESCC tissues and cell lines, particularly in ESCC tissues with lymph node metastasis. Furthermore, it was demonstrated that function of miR-106b in ESCC was dependent on regulating PTEN mediated EMT. miR-106b may be a potential therapeutic target for the treatment of ESCC.

\section{Acknowledgements}

The present study was supported by the Science and Technology Project of the Zhengzhou Municipal Science and Technology Bureau (grant no. 141PPTGG438).

\section{References}

1. Qi B, Lu JG, Yao WJ, Chang TM, Qin XG, Ji YH, Wang TY,Liu SG, Li HC, Liu YZ and Zhao BS: Downregulation of microRNA-382 is associated with poor outcome of esophageal squamous cell carcinoma. World J Gastroenterol 21: 6884-6891, 2015.

2. Li X, Wu Z, Mei Q, Li X, Guo M, Fu X and Han W: Long non-coding RNA HOTAIR, a driver of malignancy, predicts negative prognosis and exhibits oncogenic activity in oesophageal squamous cell carcinoma. Br J Cancer 109: 2266-2278, 2013.

3. Iorio MV and Croce CM: MicroRNA dysregulation in cancer: Diagnostics, monitoring and therapeutics. A comprehensive review. EMBO Mol Med 4: 143-159, 2012.

4. Schetter AJ, Okayama H and Harris CC: The Role of microRNAs in Colorectal Cancer. Cancer J 18: 244-252, 2012.

5. Tie J, Pan Y, Zhao L, Wu K, Liu J, Sun S, Guo X, Wang B, Gang Y, Zhang Y, et al: mir-218 inhibits invasion and metastasis of gastric cancer by targeting the Robol receptor. PLoS Genet 6: e1000879, 2010

6. Li X, Liu X, Xu W, Zhou P, Gao P, Jiang S, Lobie PE and Zhu T: c-MYC-regulated miR-23a/24-2/27a cluster promotes mammary carcinoma cell invasion and hepatic metastasis by targeting Sprouty2. J Biol Chem 288: 18121-18133, 2013.

7. Zhang GJ, Li JS, Zhou H, Xiao HX, Li Y and Zhou T: MicroRNA-106b promotes colorectal cancer cell migration and invasion by directly targeting DLC1. J Exp Clin Cancer Res 34: 73, 2015.

8. Yau WL, Lam CS, Ng L, Chow AK, Chan ST, Chan JY, Wo JY, Ng KT, Man K, Poon RT and Pang RW: Over-expression of miR-106b promotes cell migration and metastasis in hepatocellular carcinoma by activating epithelial-mesenchymal transition process. PLoS One 8: e57882, 2013.

9. Zhou Y, Hu Y, Yang M, Jat P, Li K, Lombardo Y, Xiong D, Coombes RC, Raguz S and Yagüe E: The miR-106b 25 cluster promotes bypass of doxorubicin-induced senescence and increase in motility and invasion by targeting the E-cadherin transcriptional activator EP300. Cell Death Differ 21: 462-474, 2014

10. Wang H, Liu J, Zong Y, Xu Y, Deng W, Zhu H, Liu Y, Ma C, Huang L, Zhang L and Qin C: miR-106b aberrantly expressed in a double transgenic mouse model for Alzheimer's disease targets TGF- $\beta$ type II receptor. Brain Res 1357: 166-174, 2010.

11. Ivanovska I, Ball AS, Diaz RL, Magnus JF, Kibukawa M, Schelter JM, Kobayashi SV, Lim L, Burchard J, Jackson AL, et al: MicroRNAs in the miR-106b family regulate $\mathrm{p} 21 / \mathrm{CDKN1A}$ and promote cell cycle progression. Mol Cell Biol 28: 2167-2174, 2008.

12. Dai F, Liu T, Zheng S, Liu Q, Yang C, Zhou J, Chen Y, Sheyhidin I and Lu X: miR-106b promotes migration and invasion through enhancing EMT via downregulation of Smad 7 in Kazakh's esophageal squamous cell carcinoma. Tumour Biol 37: 14595-14604, 2016.
13. Zheng L, Zhang Y, Liu Y, Zhou M, Lu Y, Yuan L, Zhang C, Hong $M$, Wang $S$ and Li X: miR-106b induces cell radioresistance via the PTEN/PI3K/AKT pathways and p21 in colorectal cancer. J Transl Med 13: 252, 2015.

14. Li Y, Cui J, Zhang CH, Yang DJ, Chen JH, Zan WH, Li B, Li Z and He YL: High-expression of DJ-1 and loss of PTEN associated with tumor metastasis and correlated with poor prognosis of gastric carcinoma. Int J Med Sci 10: 1689-1697, 2013.

15. Yan T, Jiang X, Guo X, Chen W, Tang D, Zhang J, Zhang X, Zhang D, Zhang Q, Jia J and Huang Y: Electric field-induced suppression of PTEN drives epithelial-to-mesenchymal transition via mTORC1 activation. J Dermatol Sci 85: 96-105, 2017.

16. Livak KJ and Schmittgen TD: Analysis of relative gene expression data using real-time quantitative PCR and the 2(-Delta Delta C(T)) method. Methods 25: 402-408, 2001.

17. Wang T, Xuan X, Li M, Gao P, Zheng Y, Zang W and Zhao G: Astragalus saponins affect proliferation, invasion and apoptosis of gastric cancer BGC-823 cells. Diagn Pathol 8: 179, 2013.

18. Harazono Y, Muramatsu T, Endo H, Uzawa N, Kawano T, Harada K, Inazawa J and Kozaki K: miR-655 is an EMT-suppressive MicroRNA targeting ZEB1 and TGFBR2. PLoS One 8: e62757, 2013.

19. Qi Q, Ling Y,Zhu M,Zhou L, Wan M, Bao Y and Liu Y: Promoter region methylation and loss of protein expression of PTEN and significance in cervical cancer. Biomed Rep 2: 653-658, 2014.

20. Wang W and Luo YP: MicroRNAs in breast cancer: Oncogene and tumor suppressors with clinical potential. J Zhejiang Univ Sci B 16: 18-31, 2015

21. Xu M and Mo YY: The Akt-associated microRNAs. Cell Mol Life Sci 69: 3601-3612, 2012

22. Mei LL, Qiu YT, Zhang B and Shi ZZ: MicroRNAs in esophageal squamous cell carcinoma: Potential biomarkers and therapeutic targets. Cancer Biomark 19: 1-9, 2017.

23. Sakai NS, Samia-Aly E, Barbera M and Fitzgerald RC: A review of the current understanding and clinical utility of miRNAs in esophageal cancer. Semin Cancer Biol 23: 512-521, 2013.

24. Hudson RS, Yi M, Esposito D, Glynn SA, Starks AM, Yang Y, Schetter AJ, Watkins SK, Hurwitz AA, Dorsey TH, et al: MicroRNA-106b-25 cluster expression is associated with early disease recurrence and targets caspase- 7 and focal adhesion in human prostate cancer. Oncogene 32: 4139-4147, 2013.

25. Li BK, Huang PZ, Qiu JL, Liao YD, Hong J and Yuan YF: Upregulation of microRNA-106b is associated with poor prognosis in hepatocellular carcinoma. Diagn Pathol 9: 226, 2014.

26. Yang Q, Zhang RW, Sui PC, He HT and Ding L: Dysregulation of non-coding RNAs in gastric cancer. World J Gastroenterol 21: 10956-10981, 2015

27. Cai K, Wang Y and Bao X: miR-106b promotes cell proliferation via targeting RB in laryngeal carcinoma. J Exp Clin Cancer Res 30: 73, 2011.

28. Ni X, Xia T, Zhao Y, Zhou W, Wu N, Liu X, Ding Q, Zha X, Sha J and Wang S: Downregulation of miR-106b induced breast cancer cell invasion and motility in association with overexpression of matrix metalloproteinase 2. Cancer Sci 105: 18-25, 2014.

29. Ma F, Li W, Liu C, Li W, Yu H, Lei B, Ren Y, Li Z, Pang D and Qian C: miR-23a promotes TGF- $\beta 1$-induced EMT and tumor metastasis in breast cancer cells by directly targeting CDH1 and activating Wnt/ $\beta$-catenin signaling. Oncotarget 8: 69538-69550, 2017.

30. Xu W, Yang Z, Zhou SF and Lu N: Posttranslational regulation of phosphatase and tensin homolog (PTEN) and its functional impact on cancer behaviors. Drug Des Devel Ther 8: 1745-1751, 2014.

31. Madhunapantula SV and Robertson GP: The PTEN-AKT3 Signaling Cascade as a Therapeutic Target in Melanoma. Pigment Cell Melanoma Res 22: 400-419, 2009.

32. Georgescu MM: PTEN tumor suppressor network in PI3K-Akt pathway control. Genes Cancer 1: 1170-1177, 2010.

33. Ren P, Gong F, Zhang Y, Jiang J and Zhang H: MicroRNA-92a promotes growth, metastasis, and chemoresistance in non-small cell lung cancer cells by targeting PTEN. Tumour Biol 37: 3215-25, 2016.

34. Zheng X, Li J, Peng C, Zhao J, Chi J, Meng X, Yun X, Li D, Yu Y, Gao M and Li Y: MicroRNA-4 induces cisplatin resistance by targeting PTEN in human tongue squamous cell carcinoma. Oral Oncol 51: 998-1003, 2015. 